\title{
Presence of Escherichia coli carrying the EAST1 toxin gene in farm animals
}

\author{
Sophie VeIlleuX, J. Daniel DubreuiL* \\ Groupe de recherche sur les maladies infectieuses du porc (GREMIP), \\ Département de pathologie et microbiologie, Faculté de médecine vétérinaire, \\ Université de Montréal, 3200 rue Sicotte, Saint-Hyacinthe, Québec, J2S 7C6, Canada
}

(Received 23 March 2005; accepted 4 July 2005)

\begin{abstract}
Enteroaggregative Escherichia coli (EAEC) heat-stable toxin 1 (EAST1) is a small toxin of proteic nature. The reference strain producing this toxin was originally detected in the stools of a diarrheic Chilean child. Today, we know that EAST1 is not solely associated with EAEC but also with many other diarrheic $E$. coli families. Some studies have established the role of EAST1 in human outbreaks of diarrhea. In addition, isolates from farm animals were shown, more recently, to carry the astA gene coding for EAST1. However, the relation between the presence of EAST1 and disease is not conclusive. In this article, the current state of the knowledge on the presence and probable role of EAST1 in farm animal diseases is reviewed and discussed.
\end{abstract}

\section{Escherichia coli / EAST1 toxin / farm animals / diarrhea / edema disease}

Table of contents

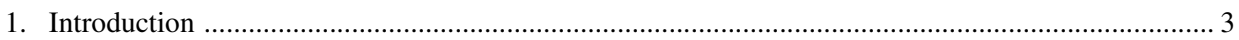

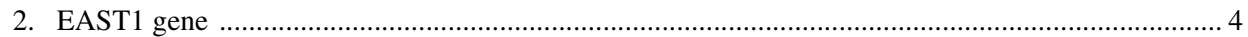

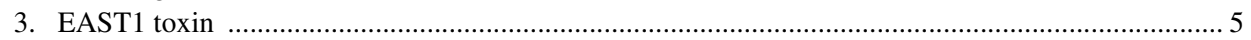

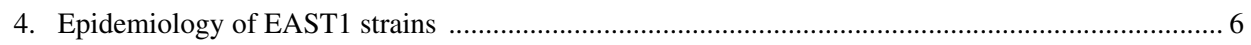

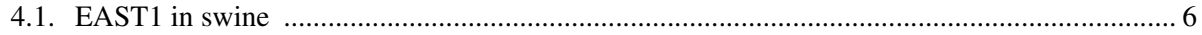

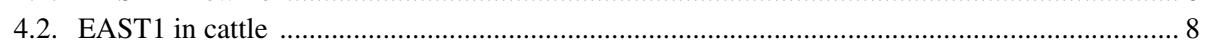

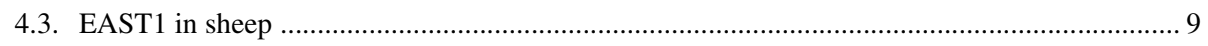

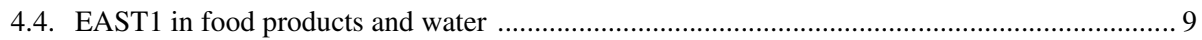

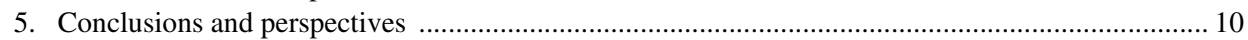

\section{INTRODUCTION}

The family of diarrheagenic Escherichia coli encompass various members including enterohemorrhagic E. coli (EHEC), enteropathogenic E. coli (EPEC), enteroinvasive
E. coli (EIEC), enterotoxigenic E. coli (ETEC), and more recently, diffusely adherent $E$. coli (DAEC) and enteroaggregative E. coli (EAEC) [25]. These E. coli are classified in different pathotypes based on the presence of specific virulence factors $[18$,

* Corresponding author: daniel.dubreuil@umontreal.ca 
41]. Each pathotype has various virulence factors that allow them to induce clinical symptoms with specific epidemiological and pathological characteristics [10, 33].

EAEC are E. coli strains that adhere in vitro to human epithelial cells (HEp-2) in a typical stacked brick pattern. EAEC strains do not produce heat-labile (LT) or heat-stable (ST) toxins [20]. EAEC were rapidly recognized as forming an important enteric pathogen group. They are responsible for diarrhea in travelers, children in the developing world and in HIV-infected patients [18]. Many EAEC outbreaks have occurred in developed and industrialized countries [18]. In some regions of the developing world, EAEC are the second most common cause of traveler's diarrhea only surpassed by ETEC. In humans, aggregative adherence fimbriae I and II (AAF-I and AAF-II), plasmid-encoded toxin (Pet) and the enteroaggregative $E$. coli heat-s stable toxin 1 (EAST1) are some of the various virulence factors associated with EAEC [23]. Recently, an E. coli strain (O166:H15) having EAST1 as the only known virulence factor has been the cause of a gastroenteritis outbreak in Osaka, Japan [50].

EAST1 was first discovered in EAEC strain 17-2 isolated from the stools of a Chilean child suffering from diarrhea [34]. EAST1 is the product of the astA gene that can be found on the chromosome and/or plasmids, in one or more copy [23]. EAST1 is a peptide of 38 amino acids with a molecular weight of $4.1 \mathrm{kDa}$. The four cysteines of the toxin are involved in the formation of two disulfide bridges [24]. Further studies associated EAST1 with E. coli pathotypes other than EAEC, such as EHEC, ETEC, DAEC and EPEC, and also with other bacterial genera like Salmonella [36]. Although clearly associated with $E$. coli strains isolated from humans, EAST1-positive strains have also been observed in farm animals. For now, it has been most commonly associated with swine and cattle. These strains are isolated from healthy or ill animals and are usually associated with symptoms like diarrhea and/or edema diseases [23]. Strain 17-2 was shown to cause diarrhea and sometimes death in a gnotobiotic piglet model [40].

An EAST1 variant, isolated from EAEC strain O-42, was observed in epidemiological studies [34]. EAST1 O-42 differs from EAST1 17-2 at codon 21 (ACA to GCA) leading to a single amino acid change ( $\mathrm{T}$ to A) [23]. In humans, this variant is more frequently isolated from diarrhea-associated E. coli strains than EAST1 17-2. Furthermore, strain O-42 seems to be more virulent than strain 17-2, which failed to induce diarrhea, when administered to volunteers [26]. This study indicated that EAST1 produced by EAEC strain O-42 could represent a potent toxic molecule whereas the toxin elaborated by strain 17-2 would be a variant with lower potency [46]. Multiple other variants of EAST1 have been isolated from sporadic cases in the context of epidemiological studies [23]. These variants seem less frequently distributed and their toxicity was not evaluated. These variants are discussed in detail in the review by Ménard and Dubreuil [23]. In fact, these variants, although described at the sequence level, were neither purified nor tested in an appropriate animal model nor in an in vitro test.

\section{EAST1 GENE}

The amino acid sequence of EAST1 was deduced from an ORF named astA, a 117bp-long DNA sequence. The $\mathrm{G}+\mathrm{C}$ content of $a s t A$ is $53 \%$, a little higher than the mean value for $E$. coli but EAST1 shows an atypically low codon adaptation index suggesting a deviation from the preferred codon usage in E. coli. The nucleotide sequences flanking astA ORF were not characterized and no promoter or regulator was identified. The gene ast $A$ has been observed in plasmids and on the chromosome of various isolates, in one or more copy. There is no homology between astA and estA, the structural gene for STa (see the review article by Ménard and Dubreuil [23]). 
Table I. Primary sequence comparison of heat-stable toxins elaborated by $E$. coli. The number of amino acids in the mature toxins is indicated in the second column. In bold is the sequence more or less conserved known to bind to guanylate cyclase $\mathrm{C}$, the receptor for STa toxin. Underlined, the cysteine residues. The asterisk indicates the change in amino acids between EAST1 variants 17-2 and $\mathrm{O}-42$.

\begin{tabular}{|c|c|c|}
\hline Toxin & Amino acids & Primary sequence \\
\hline EAST1 17-2 & 38 & MPSTQYIRRPASSYASCIWCT $*$ ACASCEHGRTTKPSLAT ${ }^{1}$ \\
\hline EAST1 O-42 & 38 & 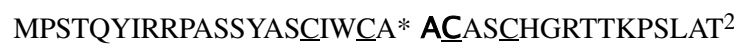 \\
\hline STaH & 19 & NSSNYCCELCCNPACTGCY ${ }^{3}$ \\
\hline STaP & 18 & NTFYCCELCCNPACAGCY ${ }^{4}$ \\
\hline
\end{tabular}

${ }^{1}$ Savarino et al. [35]; ${ }^{2}$ Yamamoto et al. [48]; ${ }^{3}$ Aimoto et al. [1]; ${ }^{4}$ Takao et al. [38].

\section{EAST1 TOXIN}

EAST1 is a 38 amino acid peptide with a calculated molecular weight of $4.1 \mathrm{kDa}$ and a pI of 9.25 (Tab. I). Unlike STa, a classic signal peptide was not observed in the $\mathrm{N}$-terminus of the predicted EAST1 sequence. Most information on this toxin was acquired through the use of culture filtrates or by direct use of EAST1-producing strains since EAST1 was purified only recently [24]. Culture filtrates from EAEC strain 17-2, heated at $65{ }^{\circ} \mathrm{C}$ for $15 \mathrm{~min}$, has demonstrated partial heat stability. Among the 38 amino acids composing EAST1, there are four cysteines at positions 17, 20, 24, and 27 , which have been shown recently to form two disulfide bridges [24]. Indirect evidence of the importance for biological activity of the disulfide bridges formed between cysteines 17 and 27 was obtained when cysteine 17 was replaced by an alanine: it resulted in inactivation of EAST1 [23]. A synthetic peptide composed of residues 8 to 29 of EAST1 induced a response in the Ussing chamber, indicating that the toxic domain was contained within this fragment [35].

EAST1 is often compared to STa, another $E$. coli heat-stable enterotoxin. Both are heat-stable toxins of low molecular weight (4.1 kDa for EAST1 and $2 \mathrm{kDa}$ for STa) where disulfide bridges are present ( 2 bridges for EAST1 and 3 for STa). Considering the pI, EAST1 $(\mathrm{pI}=9.25)$ and STa $(\mathrm{pI}=3.98)$ are peptides with a quite dissimilar net charge. EAST1 shares 50\% identity with the enterotoxigenic domain of STa (amino acid 6 to 18) [35]. Furthermore, EAST1 and STa share cGMP as a second messenger in host intestinal epithelial cells [34]. The receptor for STa toxin is guanylate cyclase $\mathrm{C}$ and the amino acid sequence determined to bind to this molecule is NPAC (Tab. I). For EAST1, two amino acids (AC) are present and it is inferred that it may be responsible for binding to the same molecule. In the same in vitro model, EAST1 induced a response in approximately $30-40 \mathrm{~min}$ as also observed for STa using rabbit ileal tissue. Savarino et al. [34] observed that under $\mathrm{Cl}^{-}$and $\mathrm{HCO}^{-}$-free conditions, the short circuit current was decreased suggesting that these ions could be involved in the electrical response. On the contrary, the reference enterotoxicity assay for STa using suckling mice (CD-1 and CFW mice) failed to elicit fluid accumulation with EAST1 [34]. However, the Giannella [12] standard STa assay was originally done using Swiss Albino mice thus, the sensitivity of the mouse strains used could probably account for the lack of biological activity observed for EAST1. In the Ussing chamber, polyclonal anti-STa antibody was inefficient in an attempt to neutralize EAST1 biological activity indicating that these toxins are immunologically different [34]. 


\section{EPIDEMIOLOGY OF EAST1 STRAINS}

EAEC affects children as well as adults from both developing and developed countries. The largest reported outbreak of diarrhea due to EAEC in human beings occurred in Japan [19]. This episode involved 16 schools with 2697 children suffering from diarrhea after consuming contaminated lunches shown to contain an EAST1-producing E. coli strain. Another study conducted in Spain has, as well, clearly shown an association between EAST1-positive strains and diarrhea in children [42].

Aggregative adherence to the intestinal mucosa is the first step in the pathogenesis of EAEC. EAST1 is a toxin that is thought to play a role in the pathogenesis of this group of bacteria although not all EAEC causing diarrhea elaborate EAST1. Numerous studies with human or animal isolates indicated that EAST1 is not restricted to EAEC [23]. The EAST1 toxin gene was not associated with porcine $E$. coli strains until 1997 [46]. Then, the EAST1 toxin was reported in epidemiological studies involving swine and cattle suffering from diarrhea and/or edema disease [31]. In general, pathogenic E. coli, except EHEC, does not originate from animals but they spread it to humans by food and water contaminated with excrements. Concerning food animals, cattle are the major reservoir of EHEC [33].

\subsection{EAST1 in swine}

The pathogenesis of EAEC infection is not fully understood but characteristic histopathological lesions and multiple virulence factor candidates were described. One of the toxins elaborated by these strains is EAST1.

Choi et al. [7] screened 720 E. coli strains, isolated from preweaning diarrheic piglets, for the presence of the astA gene using the Polymerase Chain Reaction (PCR). They evaluated the association between the EAST1 gene and the following enterotoxins: STa, STb and LT. The authors found that 164
$(22.7 \%)$ isolates carried the gene for EAST1. Moreover, 62/164 (37.8\%) isolates had EAST1 as the only known virulence factor. Among the 164 EAST1-positive strains, $46 \%$ possessed the STa gene. The major pathotypes observed in this study were, in decreasing order: EAST1, EAST1/ STa, EAST1/STa/STb, EAST1/STa/F5, EAST1/STa/F4 and EAST1/STb/F4. The strong association between EAST1 and STa observed in this study may support the idea that genes encoding these enterotoxins are linked. In this study, the proportion of ETEC strains carrying the EAST1 gene was $63 \%$ (ETEC being strains carrying the gene for at least 1 fimbrial adhesin or enterotoxin). The results indicate that the gene encoding EAST1 is widely distributed among diarrheagenic E. coli isolated from preweaning piglets.

In another study, Choi et al. [8] screened 476 E. coli strains isolated from weaned pigs suffering from diarrhea and/or edema disease for the EAST1 gene using PCR. One hundred and forty nine $(31.3 \%)$ isolates contained the EAST1 gene. Amongst these 149 EAST1-positive isolates, 66 $(44.3 \%)$ carried only the EAST1 gene. Among the EAST1-positive strains 74/149 $(49.7 \%)$ were ETEC (strains positive for at least one enterotoxin but without a fimbrial adhesion factor), or 53/149 (35.6\%) (strains with a gene for enterotoxin(s) and fimbrial adhesion factor(s)) and $10.7 \%$ were shigalike toxin producing (STEC) strains. Among the isolates known to carry genes for one or more toxins, 62 carried STa, 38 STb, 31 LT and 16 STx2e genes. The most common genotypes observed were in decreasing order: EAST1, EAST1/STa, EAST1/STa/ $\mathrm{STb} / \mathrm{LT}, \mathrm{EAST} 1 / \mathrm{STa} / \mathrm{STb}$ and EAST1/ $\mathrm{STa} / \mathrm{LT}$. This study points out the high prevalence of EAST1-positive strains in weaned pigs suffering from diarrhea and/or edema disease and the possible association between EAST1 and STa genes. Together, these studies indicated that EAST1-positive strains are associated with pre- and postweaning diarrhea as well as edema disease. However, these associations do not mean that 
EAST1 plays an actual role in all these clinical problems.

Frydendahl [11] showed that of 563 E. coli isolates associated with postweaning diarrhea and edema disease in pigs, $65.8 \%$ were EAST1-positive. All strains belonging to the $\mathrm{O} 149$ serogroup harbored EAST1, STb and LT genes. In this study, O149 was the only serogroup carrying the F4 fimbriae. A close association of astA with the F4 fimbrial marker was reported. Osek [31] analyzed 270 E. coli strains obtained from pigs with postweaning diarrhea, $46.4 \%$ of these isolates possessed ast $A$ as determined by PCR. All F4-positive $E$. coli strains from piglets with diarrhea were also EAST1-positive. F4 is a major adhesin of porcine ETEC and the association of the EAST1 coding gene with F4positive ETEC strains from piglets with diarrhea suggests that EAST1 may be a virulence marker of pathogenic isolates for these animals. Furthermore, in porcine ETEC strains, the ast $A$ and $\mathrm{F} 4$ virulence genes are carried on separate plasmids [46]. The EAST1 gene sequence from pig E. coli isolates is identical to that of strain O-42 shown to be pathogenic for human volunteers [45]. Therefore, EAST1 expressed in pig isolates may play an active role in the pathogenesis of diarrhea. In fact, this study indicated that the EAST1 gene is widely distributed among $E$. coli strains isolated from piglets with postweaning diarrhea. The toxin is closely associated with F4-positive ETEC that mainly express LTI or LTI and STb enterotoxins. The most common EAST1-positive serotype was O149:K91 and these strains were mostly LTI/STb. Noamani et al. [30] observed the ast $A$ gene and the K88ac (F4) antigen in 135 isolates from O149 ETEC strains isolated from pigs with postweaning disease. Nucleotide sequences upstream of astA from porcine and bovine ETEC strains are identical, but differ from human ETEC, DAEC and EAEC [45]. A certain consensus among flanking sequences of $E$. coli human strains exist for ast $A$ [21,47]. Nevertheless, a study by Yamamoto and Nakazawa [46] indi- cated heterogeneity in the DNA sequences between $E$. coli isolated from humans and those from animals.

As part of a study on the prevalence of AIDA-I (adhesion involved in diffuse adherence) among pigs with postweaning diarrhea or edema disease, Ha et al. [15], observed that of the 45 AIDA-I-positive isolates, only 5 possessed the EAST1 gene. Ngeleka [27] identified two new E. coli pathotypes: AIDA-I/STb/EAST1 and AIDA-I/STb. In another study, $170 \mathrm{E}$. coli strains from diarrheic piglets and 120 strains isolated from non-diarrheic piglets were studied to identify $E$. coli that have the potential to cause diarrhea in pigs [28]. The main pathotype identified from diseased animals was EAST1 (13.5\%) and from healthy animals $10.8 \%$ of the isolates were EAST1-positive. Experimentally infected piglets with one EAST1-positive strain indicated that the selected isolate attached to epithelial cells, less than the isolates with the AIDA-I pathotype, but failed to induce diarrhea. In that study, the authors concluded that the EAST1 pathotype alone is not able to induce diarrhea when given to the piglet, and so, it should not be considered as an important marker for pathogenic $E$. coli isolated from a piglet suffering from diarrhea [28]. Interestingly, the AIDA-I/STb/EAST1 and AIDA-I/STb pathotypes were isolated only from diarrheic piglets and accounted for $4.7 \%$ of the isolates. The strains of these pathotypes induced diarrhea when inoculated in newborn colostrum-deprived pigs in contrast to isolates positive only for EAST1 that did not induce diarrhea, suggesting a synergistic action between these virulence factors.

In another study on the prevalence of AIDA-I, Ha et al. [16] also observed a positive association between AIDA-I and EAST1. They screened 1002 E. coli isolates from pre-weaned pigs with diarrhea in Korea and tested for the presence of the EAST1 gene in AIDA-I-positive strains. Among the 23 AIDA-I-positive strains, $17(73.9 \%)$ were also EAST1-positive. In 
addition, AIDA/EAST1 strains also possessed STa and/or STb genes suggesting a relation between these virulence traits.

$\mathrm{Vu}-$ Khac et al. [44] analyzed 92 isolates from 14 to 28 day-old piglets that died of diarrhea. The astA gene was detected in 49 $(53.3 \%)$ of the isolates, 35 of which carried genes for enterotoxins and/or fimbriae. The major genotypes observed were $\mathrm{F} 4 / \mathrm{STb} /$ EAST1, F18/STa/STb/EAST1, STb/EAST1, F6/STa/STb/EAST1 and F18/STb/EAST1. Besides F4, the fimbrial antigen F18 is one of the most important fimbriae associated with pig postweaning diarrhea and/or edema disease. This study indicated that 10 out of 92 strains carried the gene for F18. Moreover, $37.7 \%$ of the $220 \mathrm{E}$. coli strains from 1 to 14-day-old piglets with diarrhea possessed the F4 gene and all F4-positive strains were ast $A$-positive. This is in accordance with the studies of Noamani et al. [30] and Osek [31] that detected the astA gene in $100 \%$ of Canadian and Polish F4 strains, respectively. A total of 34/37 STb-positive strains (either alone or in combination with other toxins) was also positive for EAST1. The importance of this toxin association may have an echo in the study of Berberov et al. [4] who recently demonstrated the concurrent expression of LT, STb and EAST1. This study clearly established, using an isogenic strain, that following inactivation of LT toxin production (by deletion), the development of less severe dehydrating diarrhea was observed. However, EAST1 and STb toxins together were responsible for water and electrolyte losses similar to those caused by LT. This study demonstrated that the effects of the three toxins studied were important and additive.

Recently, a study by Batisson et al. [3] has identified and characterized a new factor involved in the adhesion mechanism of attaching and effacing in E. coli in swine. The gene was coined paa for porcine attaching and effacing-associated gene. Sequence analysis of paa revealed an ORF of $753 \mathrm{bp}$ encoding a $27.6 \mathrm{kDa}$ protein with 100,52 and $49 \%$ homology with Paa of enterohemorrhagic E. coli O157:H7 strains (EDL933 and Sakai), PEB3 of Campylobacter jejuni and AcfC of Vibrio cholerae, respectively. The presence of paa and eae (a gene encoding intimin, an outer membrane protein involved in intimate attachment to host cells) sequences in the porcine $\mathrm{O} 45$ strains was highly correlated with the attaching and effacing phenotypes. The observation of three eae-positive but paa-negative porcine EPEC (PEPEC) O45 strains that were attaching and effacing-negative in pig ileal explant culture [51] provided further evidence for the importance of the paa gene in the attaching and effacing activity of $\mathrm{O} 45$ strains. The complementation of the paa mutant when performed, as expected, restored the attaching and effacing function confirming the involvement of $\mathrm{Paa}$ in PEPEC pathogenecity. The Paa protein is located on the surface of bacterial cells and chicken anti-paa antibodies were able to block the adhesion of paa-positive bacteria as determined using ex vivo pig ileal explants. More recently, a study by Harel et al. (personal communication) indicated that, of 38 ETEC strains studied, carrying the paa gene, all were ast $A$-positive and this gene was on the same plasmid as paa. A high correlation was noted between paa and astA indicating that it may play an important role in the attaching and effacing function including the observed lesions and diarrhea.

\subsection{EAST1 in cattle}

Bertin et al. [6] tested 185 isolates of STEC from healthy cattle positive for the presence of $\operatorname{stx}_{2}(n=104)$, stx $_{1}(n=18)$ or both toxins $(n=63)$. They analyzed by PCR 167 st $_{2}$-positive and 18 st $_{1}$-positive isolates for the presence of astA. The EAST1 gene was poorly present among the stx $x_{2}$ positive isolates $(6.5 \%)$ and was present in $50 \%$ of the $s t x_{1}$-positive strains. Girardeau et al. [14] screened 77 E. coli afa-8 (encoding an afimbrial adhesin) isolates from farm animals (mostly bovine) and humans to compare their virulence traits. They found that the EAST1 gene was more prevalent in the isolates of the bacteremia-associated 
group. Their results showed a significant prevalence of the EAST1 gene and its association with $\operatorname{clp} G$ (the gene encoding the major subunit of CS31A) among extraintestinal afa- 8 isolates. The zoonotic potential of certain clones and the high prevalence of multiple antibiotic resistance among bovine $a f a-8$ isolates suggested that such strains could present a risk to public health. High prevalence of EAST1 toxin was also demonstrated in bovine CS31A-positive isolates [5]. It is noteworthy that bovine CS31A and porcine F4 are closely related fimbrial structures [13].

Stephan et al. [37] reported the first isolation of O157:H45 EPEC strains from cattle. The gene encoding EAST1 was found in 10 of the 11 strains studied. Cattle seem to be a reservoir of O157:H45 EPEC strains, which are described in association with human diseases. These strains could, in some instances, play a role as food-borne pathogens.

\subsection{EAST1 in sheep}

Cookson et al. [9] experimentally inoculated four lambs at 6 weeks of age with an E. coli $\mathrm{O} 157: \mathrm{H} 7$. Attaching-effacing lesions were observed in the caecum, proximal colon and rectum of one lamb. The attached bacteria did not immunostain with the O157-specific antiserum. From this animal, the bacteriological analysis of samples yielded two isolates $\mathrm{O} 115: \mathrm{H}(-)$, found to form microcolonies and attaching and effacing lesions on Hep-2 cells. Supernatants of both $\mathrm{O} 115: \mathrm{H}(-)$ isolates induced cytopathic effects on Vero cell monolayers. Both isolates encoded EAST1, CNF1 (cytotoxic necrotizing factor) and CNF2 toxins as determined by PCR analysis. These $E$. coli strains exhibited a novel combination of virulence determinants and are the first isolates found to possess both CNF1 and CNF2.

\subsection{EAST1 in food products and water}

Since swine, cattle and sheep are food animals, the presence of EAST1 toxin in bacterial isolates from these animals may result in a transmission to humans through the food chain. Toshima et al. [39] conducted a study on food heavily contaminated with coliforms (115 samples). Using PCR, they observed the highest prevalence of the EAST1 gene in animal products tested (16/ $54,29.6 \%)$. On the contrary, EAST1 was rarely found in foods of plant origin (2/45) or fishery products (1/16). Although EAST1 was unexpectedly common in animal products, its potential as a human pathogen remains uncertain since some virulence properties differ significantly between strains from fecal and food isolates. Some food isolates, however, possess the same virulence characteristics as fecal isolates.

A few food-borne diarrhea outbreaks caused by atypical diarrheagenic E. coli strains involving adults have been reported. One outbreak in the USA involving more than 100 adults, patrons of the same restaurant, implicated an E. coli O39:NM [17]. This strain was LT, STa and STb-negative. Another diarrhea outbreak in Finland involving 650 humans (611 students and 39 adults in a school) was reported [43]. The pathogen responsible for this outbreak was an E. coli O111:B4. Interestingly, both $E$. coli responsible for the food-borne outbreaks were astA-positive.

In addition to food products, water contaminated by animal feces could also represent, in some regions of the world including industrialized countries, a way of transmission to human beings. For example, E. coli strains were associated with a waterborne diarrhea outbreak among students and teachers attending a fieldwork program held at a farm in the Akita prefecture, Japan [49]. Among the 75 attendees, 41 students showed clinical signs including diarrhea ( $85 \%$ of patients). A marked difference in the attack rate was noted between students who drank tap water $(86.1 \%)$ at the pasture and those who only washed their hands $(26.3 \%)$. The tap water was provided from a brook but without chlorination. Seven E. coli Ouk $: \mathrm{H} 45$ isolates were 
recovered from the patients as the causative agent of the outbreak. One isolate was attaching and effacing (eae) and astA-positive. In this study, one strain, EC-3605 represented an atypical EPEC strain harboring astA. The role that atypical EPEC strains harboring the astA gene play in human disease is unclear.

These food-borne and water-borne diarrhea outbreaks in humans illustrate the problem that can be encountered when contamination from $E$. coli elaborating toxins such as EAST1 are associated with farm animals. However, in most epidemiological studies, evidence of such cross-contamination has not been published for EAEC that are EAST1-positive.

\section{CONCLUSIONS AND PERSPECTIVES}

For now, the pathogenicity of E. coli strains producing EAST1 only has not been determined in an animal model under rigorous experimental conditions. Studies on the correlation between the presence of the astA gene and expression of active EAST1 toxin are also needed because PCR studies indicate the presence of astA but without certifying the synthesis of an active toxin. The oligonucleotide primers used in the reported studies target EAST1 17-2 and at best both variants 17-2 and O-42. Most of the time, variants of EAST1 other than 17-2 and $\mathrm{O}-42$ are ignored suggesting that EAST1 is probably present more often than actually determined by PCR. Further studies may be needed to define a role of EAST1 in the pathogenesis of pig and cattle diarrhea. On the contrary to what was determined for human EAST1-positive strains, there is no clear evidence of EAST1dependent fluid secretion in animal studies [22] or of an association of EAST1 with epidemic strains of $E$. coli with high diarrheagenic potential. The late purification of EAST1 toxin [24] by our group could provide a source of pure toxin in order to test if diarrhea could result from the administration of pure toxin to animals without the cumbersome interpretation that we encounter when using $E$. coli isolates or crude culture supernatants. Another challenge for future studies will be to investigate if the numerous variants already reported for EAST1 toxin are actually toxic for farm animals and also to determine their association with diarrhea outbreaks occurring in farm animals. Challenging the suckling mouse assay, the standard STa assay for the determination of the biological activity, with EAST1 could provide an answer to this question. Since the already tested CD-1 and CFW mouse strains did not indicate toxicity for purified EAST1 from strain 17-2, Swiss albino mice as used by Giannella [12] in his reference assay, should be tested for comparison purposes [24]. Ussing chambers using rabbit ileal tissue could also represent another alternative test for toxicity [34].

A study of Bacciu et al. [2] indicated that an intergeneric transfer of virulence genes (involving the astA gene) via an insertion sequence (IS1414, encoding a transposase (tnpA) and astA in Salmonella) from E. coli together with studies reporting the presence of $a s t A$ in bacterial genera besides $E$. coli, like Salmonella Agona [32] and Klebsiella pneumoniae [29] clearly indicate that horizontal transfer of virulence factors, including the ast $A$ gene, between bacterial genera occurs in nature and can account for the considerable distribution of the EAST1 gene in $E$. coli besides EAEC. Yet, there is no evidence of EAST1-dependent fluid secretion in animal studies or of an association of EAST1 with epidemic strains of $E$. coli with high diarrheagenic potential [2].

\section{ACKNOWLEDGEMENTS}

The authors are financially partly supported by the Canadian Research Network on Bacterial Pathogens of Swine. 


\section{REFERENCES}

[1] Aimoto S., Takao T., Shimonishi Y., Hara S., Takeda T., Takeda Y., Miwatani T., Aminoacid sequence of a heat-stable enterotoxin produced by human enterotoxigenic Escherichia coli, Eur. J. Biochem. 129 (1982) 257-263.

[2] Bacciu D., Falchi G., Spazziani A., Bossi L., Marogna G., Leori G.S., Rubino S., Uzzau S., Transposition of the heat-stable toxin astA gene into a gifsy-2-related prophage of Salmonella enterica serovar Abortusovis, J. Bacteriol. 186 (2004) 4568-4574.

[3] Batisson I., Guimond M.P., Girard F., An H., Zhu C., Oswald E., Fairbrother J.M., Jacques M., Harel J., Characterization of the novel factor paa involved in the early steps of the adhesion mechanism of attaching and effacing Escherichia coli, Infect. Immun. 71 (2003) 4516-4525.

[4] Berberov E.M., Zhou Y., Francis D.H., Scott M.A., Kachman S.D., Moxley R.A., Relative importance of heat-labile enterotoxin in the causation of severe diarrheal disease in the gnotobiotic piglet model by a strain of enterotoxigenic Escherichia coli that produces multiple enterotoxins, Infect. Immun. 72 (2004) 3914-3924.

[5] Bertin Y., Martin C., Girardeau J.P., Pohl P., Contrepois M., Association of genes encoding $\mathrm{P}$ fimbriae, CS31A antigen and EAST 1 toxin among CNF1-producing Escherichia coli strains from cattle with septicemia and diarrhea, FEMS Microbiol. Lett. 162 (1998) 235-239.

[6] Bertin Y., Boukhors K., Pradel N., Livrelli V., Martin C., Stx2 subtyping of Shiga toxin-producing Escherichia coli isolated from cattle in France: detection of a new Stx2 subtype and correlation with additional virulence factors, J. Clin. Microbiol. 39 (2001) 3060-3065.

[7] Choi C., Cho W., Chung H., Jung T., Kim J., Chae C., Prevalence of the enteroaggregative Escherichia coli heat-stable enterotoxin 1 (EAST1) gene in isolates in weaned pigs with diarrhea and/or edema disease, Vet. Microbiol. 81 (2001) 65-71.

[8] Choi C., Kwon D., Chae C., Prevalence of the enteroaggregative Escherichia coli heat-stable enterotoxin 1 gene and its relationship with fimbrial and enterotoxin genes in $E$. coli isolated from diarrheic piglets, J. Vet. Diagn. Invest. 13 (2001) 26-29.

[9] Cookson A.L., Hayes C.M., Pearson G.R., Roe J.M., Wales A.D., Woodward M.J., Isolation from a sheep of an attaching and effacing Escherichia coli O115:H- with a novel combination of virulence factors, J. Med. Microbiol. 51 (2002) 1041-1049.

[10] Fasano A., Cellular microbiology: can we learn cell physiology from microorganisms? Am. J. Physiol. 276 (1999) C765-C776.

[11] Frydendahl K., Prevalence of serogroups and virulence genes in Escherichia coli associated with postweaning diarrhoea and edema disease in pigs and a comparison of diagnostic approaches, Vet. Microbiol. 85 (2002) 169182.

[12] Giannella R.A., Suckling mouse model for detection of heat-stable Escherichia coli enterotoxin: characteristics of the model, Infect. Immun. 14 (1976) 95-99.

[13] Girardeau J.P., Der Vartanian M., Ollier J.L., Contrepois M., CS31A, a new K88-related fimbrial antigen on bovine enterotoxigenic and septicemic Escherichia coli strains, Infect. Immun. 56 (1988) 2180-2188.

[14] Girardeau J.P., Lalioui L., Said A.M., De Champs C., Le Bouguenec C., Extended virulence genotype of pathogenic Escherichia coli isolates carrying the afa- 8 operon: evidence of similarities between isolates from humans and animals with extraintestinal infections, J. Clin. Microbiol. 41 (2003) 218226.

[15] Ha S.K., Choi C., Chae C., Prevalence of a gene encoding adhesin involved in diffuse adherence among Escherichia coli isolates in pigs with postweaning diarrhea or edema disease, J. Vet. Diagn. Invest. 15 (2003) 378381.

[16] Ha S.K., Choi C., Jung K., Kim J., Han D.U., Ha Y., Lee S.D., Kim S.H., Chae C., Genotypic prevalence of the adhesin involved in diffuse adherence in Escherichia coli isolates in pre-weaned pigs with diarrhoea in Korea, J. Vet. Med. B 51 (2004) 166-168.

[17] Hedberg C.W., Savarino S.J., Besser J.M., Paulus C.J., Thelen V.M., Myers L.J., Cameron D.N., Barrett T.J., Kaper J.B., Osterholm M.T., An outbreak of foodborne illness caused by Escherichia coli O39:NM, an agent not fitting into the existing scheme for classifying diarrheogenic E. coli, J. Infect. Dis. 176 (1997) 1625-1628.

[18] Huang D.B., Okhuysen P.C., Jiang Z.D., DuPont H.L., Enteroaggregative Escherichia coli: an emerging enteric pathogen, Am. J. Gastroenterol. 99 (2004) 383-389.

[19] Itoh Y., Nagano I., Kunishima M., Ezaki T., Laboratory investigation of enteroaggregative Escherichia coli $\mathrm{O}$ untypeable:H10 associated with a massive outbreak of gastrointestinal illness, J. Clin. Microbiol. 35 (1997) 2546-2550. 
[20] Kaper J.B., Nataro J.P., Mobley H.L., Pathogenic Escherichia coli, Nat. Rev. Microbiol. 2 (2004) 123-140.

[21] Lalioui L., Jouve M., Gounon P., Le Bouguenec C., Molecular cloning and characterization of the afa-7 and $a f a-8$ gene clusters encoding afimbrial adhesins in Escherichia coli strains associated with diarrhea or septicemia in calves, Infect. Immun. 67 (1999) 5048-5059.

[22] McVeigh A., Fasano A., Scott D.A., Jelacic S., Moseley S.L., Robertson D.C., Savarino S.J., IS1414, an Escherichia coli insertion sequence with a heat-stable enterotoxin gene embedded in a transposase-like gene, Infect. Immun. 68 (2000) 5710-5715.

[23] Ménard L.P., Dubreuil J.D., Enteroaggregative Escherichia coli heat-stable enterotoxin 1 (EAST1): a new toxin with an old twist, Crit. Rev. Microbiol. 28 (2002) 43-60.

[24] Ménard L.P., Lussier J.G., Lépine F., Paiva de Sousa C., Dubreuil J.D., Expression, purification, and biochemical characterization of enteroaggregative Escherichia coli heat-stable enterotoxin 1, Protein Expr. Purif. 33 (2004) 223-231.

[26] Nataro J.P., Kaper J.B., Diarrheagenic Escherichia coli, Clin. Microbiol. Rev. 11 (1998) 142-201.

[25] Nataro J.P., Deng Y., Cookson S., Cravioto A., Savarino S.J., Guers L.D., Levine M.M., Tacket C.O., Heterogeneity of enteroaggregative Escherichia coli virulence demonstrated in volunteers, J. Infect. Dis. 171 (1995) 465468.

[27] Ngeleka M., Isolation of a new Escherichia coli pathotype associated with diarrhea in piglets, Can. Vet. J. 43 (2002) 623-624.

[28] Ngeleka M., Pritchard J., Appleyard G., Middleton D.M., Fairbrother J.M., Isolation and association of Escherichia coli AIDA-I/ $\mathrm{STb}$, rather than EAST1 pathotype, with diarrhea in piglets and antibiotic sensitivity of isolates, J. Vet. Diagn. Invest. 15 (2003) 242 252.

[29] Nguyen Thi P.L., Yassibanda S., Aidara A., Le Bouguenec C., Germani Y., Enteropathogenic Klebsiella pneumoniae HIV-infected adults, Africa, Emerg. Infect. Dis. 9 (2003) 135-137.

[30] Noamani B.N., Fairbrother J.M., Gyles C.L., Virulence genes of O149 enterotoxigenic Escherichia coli from outbreaks of postweaning diarrhea in pigs, Vet. Microbiol. 97 (2003) $87-101$.
[31] Osek J., Detection of the enteroaggregative Escherichia coli heat-stable enterotoxin 1 (EAST1) gene and its relationship with fimbrial and enterotoxin markers in E. coli isolates from pigs with diarrhoea, Vet. Microbiol. 91 (2003) 65-72.

[32] Paiva de Sousa C., Dubreuil J.D., Distribution and expression of the astA gene (EAST1 toxin) in Escherichia coli and Salmonella, Int. J. Med. Microbiol. 291 (2001) 15-20.

[33] Robins-Browne R.M., Bordun A.M., Tauschek M., Bennett-Wood V.R., Russell J., Oppedisano F., Lister N.A., Bettelheim K.A., Fairley C.K., Sinclair M.I., Hellard M.E., Escherichia coli and community-acquired gastroenteritis, Melbourne, Australia, Emerg. Infect. Dis. 10 (2004) 1797-1805.

[34] Savarino S.J., Fasano A., Robertson D.C., Levine M.M., Enteroaggregative Escherichia coli elaborate a heat-stable enterotoxin demonstrable in an in vitro rabbit intestinal model, J. Clin. Invest. 87 (1991) 1450-1455.

[35] Savarino S.J., Fasano A., Watson J., Martin B.M., Levine M.M., Guandalini S., Guerry P., Enteroaggregative Escherichia coli heat-stable enterotoxin 1 represents another subfamily of $E$. coli heat-stable toxin, Proc. Natl. Acad. Sci. USA 90 (1993) 3093-3097.

[36] Savarino S.J., McVeigh A., Watson J., Cravioto A., Molina J., Echeverria P., Bhan M.K., Levine M.M., Fasano A., Enteroaggregative Escherichia coli heat-stable enterotoxin is not restricted to enteroaggregative E. coli, J. Infect. Dis. 173 (1996) 1019-1022.

[37] Stephan R., Borel N., Zweifel C., Blanco M., Blanco J.E., First isolation and further characterization of enteropathogenic Escherichia coli (EPEC) O157:H45 strains from cattle, BMC Microbiol. 4 (2004) 10.

[38] Takao T., Hitouji T., Aimoto S., Shimonishi Y., Hara S., Takeda T., Takeda Y., Miwatani T., Amino acid sequence of a heat-stable enterotoxin isolated from enterotoxigenic Escherichia coli strain 18D, FEBS Lett. 152 (1983) $1-5$.

[39] Toshima H., Uenaka E., Bi Y., Nakamura H., Ogasawara J., Hase A., Kamata Y., Nishikawa Y., Detection and isolation of Escherichia coli with a coding gene for enteroaggregative Escherichia coli heat-stable enterotoxin 1 from food and comparison with fecal isolates, J. Food Prot. 67 (2004) 2117-2122.

[40] Tzipori S., Montanaro J., Robins-Browne R.M., Vial P., Gibson R., Levine M.M., Studies with enteroaggregative Escherichia coli in the gnotobiotic piglet gastroenteritis model, Infect. Immun. 60 (1992) 5302-5306. 
[41] Uzzau S., Fasano A., Cross-talk between enteric pathogens and the intestine, Cell Microbiol. 2 (2000) 83-89.

[42] Vila J., Gene A., Vargas M., Gascon J., Latorre C., Jimenez de Anta M.T., A case-control study of diarrhoea in children caused by Escherichia coli producing heat-stable enterotoxin (EAST-1), J. Med. Microbiol. 47 (1998) 889-891.

[43] Viljanen M.K., Peltola T., Junnila S.Y., Olkkonen L., Jarvinen H., Kuistila M., Huovinen P., Outbreak of diarrhoea due to Escherichia coli O111:B4 in schoolchildren and adults: association of $\mathrm{Vi}$ antigen-like reactivity, Lancet 336 (1990) 831-834.

[44] Vu-Khac H., Holoda E., Pilipcinec E., Distribution of virulence genes in Escherichia coli strains isolated from diarrhoeic piglets in the Slovak Republic, J. Vet. Med. B 51 (2004) 343-347.

[45] Yamamoto T., Echeverria P., Detection of the enteroaggregative Escherichia coli heat-stable enterotoxin 1 gene sequences in enterotoxigenic $E$. coli strains pathogenic for humans, Infect. Immun. 64 (1996) 1441-1445.

[46] Yamamoto T., Nakazawa M., Detection and sequences of the enteroaggregative Escherichia coli heat-stable enterotoxin 1 gene in enterotoxigenic $E$. coli strains isolated from piglets and calves with diarrhea, J. Clin. Microbiol. 35 (1997) 223-227.
[47] Yamamoto T., Taneike I., The sequences of enterohemorrhagic Escherichia coli and Yersinia pestis that are homologous to the enteroaggregative $E$. coli heat-stable enterotoxin gene: cross-species transfer in evolution, FEBS Lett. 472 (2000) 22-26.

[48] Yamamoto T., Wakisaka N., Sato F., Kato A., Comparison of the nucleotide sequence of enteroaggregative Escherichia coli heat-stable enterotoxin 1 genes among diarrhea-associated Escherichia coli, FEMS Microbiol. Lett. 147 (1997) 89-95.

[49] Yatsuyanagi J., Saito S., Miyajima Y., Amano K., Enomoto K., Characterization of atypical enteropathogenic Escherichia coli strains harboring the astA gene that were associated with a waterborne outbreak of diarrhea in Japan, J. Clin. Microbiol. 41 (2003) 2033-2039.

[50] Zhou Z., Ogasawara J., Nishikawa Y., Seto Y., Helander A., Hase A., Iritani N., Nakamura H., Arikawa K., Kai A., Kamata Y., Hoshi H., Haruki K., An outbreak of gastroenteritis in Osaka, Japan due to Escherichia coli serogroup O166:H15 that had a coding gene for enteroaggregative E. coli heat-stable enterotoxin 1 (EAST1), Epidemiol. Infect. 128 (2002) 363-371.

[51] Zhu C., Harel J., Jacques M., Fairbrother J.M., Interaction with pig ileal explants of Escherichia coli $\mathrm{O} 45$ isolates from swine with postweaning diarrhea, Can. J. Vet. Res. 59 (1995) 118-123. 